\title{
Investigation of Dominant States in Dielectronic Recombination Rates for Fe-Ions
}

\author{
H. Ramadan, A. Khazbak, and Ali H. Moussa \\ Physics Department, Faculty of Science, Ain Shams University, Cairo, Egypt \\ Reprint requests to Dr. H. R.; E-mail: Ramadan_Hassan@hotmail.com
}

Z. Naturforsch. 58a, 346 - 350 (2003); received April 4, 2002

\begin{abstract}
Dielectronic recombination (DR) cross sections and rate coefficients are calculated for the isonuclear sequence $\mathrm{Fe}^{Z+}$, with $Z=21,20,19,18,17$, and 16, in which L-shell (2p-) excitations are involved during the initial capture. Most of the dominant transitions with $\Delta n \neq 0$, using angular momentum average (AMA) approximation, are considered. It is found that the states 3 pnd and $3 \mathrm{dnd}$ contribute most to the rate coefficients. In addition, the rates are found to increase with increasing number of electrons in the ion, (i.e. as $Z$ decreases). Moreover, the rate coefficients, $\alpha^{\mathrm{DR}}$, for the studied ions are found to peak around the same energy $(k T=30 \mathrm{Ry})$. A semi-empirical formula for the total rates $\alpha$ is obtained for the 2p-excitation with $\Delta n \neq 0$ in the case of $\mathrm{Fe}^{Z+}$ ions. On comparing both results, the explicit calculations and the results obtained from the semiempirical formula, good agreement is found. The available results for $\alpha^{\mathrm{DR}}$ may be considered as a database for future comparison with experimental and theoretical calculations. Comparison of our results with other results show the effect of the empirical rate formula.
\end{abstract}

Key words: Electron-Ion Collision; Resonance States; Auger Rates; Radiative Rates; Dielectric Recombination.

\section{Introduction}

In electron-ion collisions, the electron may be captured by a positive ion causing excitation of the bound state electrons. This process leads to the formation of doubly-excited resonance d-states. These d-states are stabilized either by the emission of X-rays or Auger electrons. This process is known as dielectronic recombination (DR). The emission of X-rays in the DR process causes self-cooling of a thermal plasma, which makes it of special interest in theoretical and experimental studies. In addition, the DR process, together with other recombination mechanisms, such as radiative recombination (RR), are responsible for the ionization balance in hot astrophysical and laboratory plasmas.

Complete calculations of DR rate coefficients for a given isonuclear sequence are often lengthy, due to the multi-step nature of the DR process. Free electron capture to double infinitely intermediate (resonant) states has to be incorporated. Further complications arise when these resonance states are decaying to final states which are themselves unstable against further Auger emission (cascade effect). Mainly because of the large amount of data involved, and the difficulty in calculating them, for a rapid generation of the data simple empirical formulas were constructed.

Burgess [1] proposed a phenomenological formula for ions of $Z_{\mathrm{c}} \leq 20$ where, at low temperature, the $\Delta n=0$ process dominates. Merts [2] later modified the formula to incorporate transitions which are important for $Z_{\mathrm{c}} \geq 15$ and $Z_{\mathrm{c}} \geq 10$. Hahn [3], based on a limited set of benchmark calculations, proposed an improved formula. Moreover, Hahn [4] has done another improvement for cases, where there is a lack of data in the region $5 \leq N \leq 9$. Moussa and Hahn [5] obtained a new empirical formula for the total rate coefficients for two transitions $(\Delta n=0$ and $\Delta n \neq 0)$ separately in the case of Ar ions. In the present work we present an improved semi-empirical formula for the $\mathrm{DR}$ rates of ions of the $\mathrm{Fe}$ isonuclear sequence. The improved formula is applied to calculate rate coefficients. As a contribution of the resonant capture process DR in electron-ion collisions [6] we examine the DR involving $\mathrm{Fe}^{Z+}$ isonuclear target ions at intermediate energies, $Z=5-10$, where $\Delta n \neq 0$ transitions are dominant.

These calculations are performed for two reasons, first, to genereate by extrapolation a complete set of $\mathrm{DR}$ rates for all ions of the $\mathrm{Fe}^{\mathrm{Z}+}$ isonuclear sequence 
and to obtain a semi-empirical formula. Second, because of the lack of available data in the region $5 \leq N \leq 9$.

\section{Theory}

Dielectronic recombination is a two step process $[7,8]$ in which a free electron is captured by a positive ion in its initial state (i) and gives rise to any one of a number of narrowly resonant, doubly excited intermediate states (d). This intermediate state then stabilizes itself by emitting a photon, and the resulting ion becomes more stable in the final state (f). The processes of interest here are described as

$$
\mathrm{e}^{-}+\mathrm{Fe}^{Z+} \rightarrow\left(\mathrm{Fe}^{(Z-1)+}\right)^{* *} \rightarrow\left(\mathrm{Fe}^{(Z-1)+}\right)^{*}
$$

where $Z$ is the initial degree of ionization. The initial states are $1 \mathrm{~s}^{2} 2 \mathrm{~s}^{2} 2 \mathrm{p}, 1 \mathrm{~s}^{2} 2 \mathrm{~s}^{2} 2 \mathrm{p}^{2}, 1 \mathrm{~s}^{2} 2 \mathrm{~s}^{2} 2 \mathrm{p}^{3}, 1 \mathrm{~s}^{2} 2 \mathrm{~s}^{2} 2 \mathrm{p}^{4}$, $1 s^{2} 2 s^{2} 2 p^{5}$, and $1 s^{2} 2 s^{2} 2 p^{6}$ for $\mathrm{Fe}^{21+}, \mathrm{Fe}^{20+}, \mathrm{Fe}^{19+}$, $\mathrm{Fe}^{18+}, \mathrm{Fe}^{17+}$, and $\mathrm{Fe}^{16+}$, respectively. The cross section is defined [8] in the initial resonance approximation by

$$
\sigma^{\mathrm{DR}}=\frac{4 \pi \tau_{0}}{e_{\mathrm{c}}(\mathrm{Ry})} V_{a}(\mathrm{i} \rightarrow \mathrm{d}) \omega(\mathrm{d} \rightarrow \mathrm{f}) \tilde{\delta}\left(e_{\mathrm{c}}, E_{\mathrm{d}}\right)\left(\pi a_{0}^{2}\right)
$$

where

$$
V_{a}(\mathrm{i} \rightarrow \mathrm{d})=\frac{g_{\mathrm{d}}}{2 g_{\mathrm{i}}} \sum_{l_{\mathrm{c}}} A_{a}\left(\mathrm{~d} \rightarrow \mathrm{i}, \ell_{\mathrm{c}}\right)
$$

is the initial excitation capture probability (in $\mathrm{s}^{-1}$ ), $g_{\mathrm{d}}$ and $g_{i}$ are the statistical weights for the intermediate and initial states, respectively, and

$$
\omega(\mathrm{d})=\frac{\Gamma_{r}(\mathrm{~d})}{\Gamma(\mathrm{d})}
$$

is the fluorescence yield, where

$$
\begin{gathered}
\Gamma(\mathrm{d})=\sum_{i} A_{a}(\mathrm{~d} \rightarrow \mathrm{i})+\sum_{r} A_{r}(\mathrm{~d} \rightarrow \mathrm{f}) \\
=\Gamma_{a}(\mathrm{~d})+\Gamma_{r}(\mathrm{~d}) \\
\tilde{\delta}\left(e_{\mathrm{c}}, E_{\mathrm{d}}\right)=\frac{\Gamma}{2 \pi} \frac{1}{\left(e_{\mathrm{c}}-E_{\mathrm{d}}\right)^{2}+\Gamma^{2} / 4}
\end{gathered}
$$

and

$$
\int \tilde{\delta} \mathrm{d} e_{\mathrm{c}}=1
$$

where $A_{a}$ and $A_{r}$ are the Auger and radiative transition probabilities, respectively. The total cross section is defined from (2) as

$$
\bar{\sigma}^{\mathrm{DR}}(\mathrm{i})=\sum \sigma^{\mathrm{DR}}(\mathrm{i} \rightarrow \mathrm{d} \rightarrow \mathrm{f}) .
$$

These quantities are evaluated using the nonrelativistic Hartree-Fock wavefunctions and angular momentum average (AMA) coupling approximation. To simplify the presentation of $\sigma^{\mathrm{DR}}$, we further define an energyaveraged cross section over a bin $\Delta e_{\mathrm{c}}$ as

$$
\bar{\sigma}^{\mathrm{DR}}=\frac{1}{\Delta e_{\mathrm{c}}} \int_{e_{\mathrm{c}}-\Delta e_{\mathrm{c}} / 2}^{e_{\mathrm{c}}+\Delta e_{\mathrm{c}} / 2} \sigma^{\mathrm{DR}}\left(e_{\mathrm{c}}^{\prime}\right) \mathrm{d} e_{\mathrm{c}}^{\prime}
$$

where $\Delta e_{\mathrm{c}}$ is arbitrarily chosen. We chose $\Delta e_{\mathrm{c}}=1 \mathrm{Ry}$ throughout this paper. The atomic time is $\tau_{0}=2.42 \times$ $10^{-17} \mathrm{sec}$.

In general, there are many intermediate resonance states (d), in fact a double infinity of states, which contribute to the total cross section $\sigma^{\mathrm{DR}}$ and the rate coefficients $\alpha^{\mathrm{DR}}$. However, previous experience has shown that only a small number $(100-200)$ of lowlying states require careful study. Moreover, contributions from the rest of states can then be estimated using the $n$ - and $Z$-scaling properties of $A_{\mathrm{a}}$ and $A_{\mathrm{r}}$ [8]. In the present case, the dominant states for the isonuclear sequence of $\mathrm{Fe}$ are investigated.

The DR rate coefficients $\alpha^{\mathrm{DR}}$ from an initial state (i) of the recombining ion to a stabilized final state (f) via an intermediate autoionizing state (d) can be expressed as

$$
\begin{aligned}
\alpha^{\mathrm{DR}}(\mathrm{i} \rightarrow \mathrm{d})= & \left(\frac{4 \pi \mathrm{Ry}}{k T}\right)^{3 / 2} \\
& \cdot a_{0}^{3} \sum_{d} \exp \left(-e_{\mathrm{c}} / k T\right) V_{a}(\mathrm{i} \rightarrow \mathrm{d}) \omega(\mathrm{d}) .
\end{aligned}
$$

Ry is the Rydberg energy and $a_{0}$ is the Bohr radius. The DR rates are in units of $\mathrm{cm}^{3} / \mathrm{s}$. $e_{\mathrm{c}}$ is the Auger energy and $T$ is the plasma electron temperature. The total rate coefficient can be obtained from (5) by summing over d- and f-states.

The detailed Auger and radiative rates and transition energies required for the evaluation of $\alpha^{\mathrm{DR}}$ were calculated explicitly for each autoionizing state. The Auger transition probability is calculated [9] using the angular momentum average scheme,

$$
A_{\mathrm{a}}(\mathrm{d} \rightarrow \mathrm{i})=\frac{4 \pi^{2}}{h}\left|\left\langle\psi_{a} \psi_{\mathrm{b}}\left|\frac{1}{r_{12}}\right| \psi_{\mathrm{g}} \psi_{\mathrm{c}}\right\rangle\right|^{2},
$$


Table 1. The DR cross sections (in $10^{-21} \mathrm{~cm}^{2}$ ) for $\mathrm{Fe}^{Z+}$-ions versus of the energy in Ry.

\begin{tabular}{|c|c|c|c|c|c|c|c|c|c|c|c|c|}
\hline \multicolumn{2}{|c|}{$\mathrm{Fe}^{21+}$} & \multicolumn{2}{|c|}{$\mathrm{Fe}^{20+}$} & \multicolumn{2}{|c|}{$\mathrm{Fe}^{19+}$} & \multicolumn{2}{|c|}{$\mathrm{Fe}^{18+}$} & \multicolumn{2}{|c|}{$\mathrm{Fe}^{17+}$} & \multicolumn{3}{|c|}{$\mathrm{Fe}^{16+}$} \\
\hline State & $e_{\mathrm{c}}$ & $\sigma^{\mathrm{DR}}$ & $e_{\mathrm{c}}$ & $\sigma^{\mathrm{DR}}$ & $e_{\mathrm{c}}$ & $\sigma^{\mathrm{DR}}$ & $e_{\mathrm{c}}$ & $\sigma^{\mathrm{DR}}$ & $e_{\mathrm{c}}$ & $\sigma^{\mathrm{DR}}$ & $e_{\mathrm{c}}$ & $\sigma^{\mathrm{DR}}$ \\
\hline $3 \mathrm{~s} 3 \mathrm{p}$ & 23.2 & 2.99 & 23.0 & 7.16 & 22.9 & 8.45 & 22.6 & 10.2 & 22.4 & 12.3 & 22.2 & 14.4 \\
\hline $3 s 4 p$ & 46.5 & 1.61 & 44.7 & 3.64 & 42.9 & 4.5 & 41.1 & 5.63 & 39.3 & 7.47 & 37.5 & 9.02 \\
\hline $3 \mathrm{~s} 5 \mathrm{p}$ & 56.6 & 1.00 & 53.9 & 2.30 & 51.3 & 3.02 & 48.8 & 5.91 & 46.3 & 5.02 & 43.8 & 6.23 \\
\hline $3 s 6 p$ & 61.9 & 0.69 & 58.8 & 1.64 & 55.8 & 2.18 & 52.8 & 2.93 & 49.9 & 3.69 & 47.0 & 4.51 \\
\hline $3 \mathrm{~s} 7 \mathrm{p}$ & 65.0 & 0.49 & 61.7 & 1.16 & 58.4 & 1.65 & 55.2 & 2.21 & 52.0 & 2.78 & 48.9 & 3.38 \\
\hline $3 \mathrm{~s} 8 \mathrm{p}$ & 67.1 & 0.36 & 63.5 & 1.16 & 60.1 & 1.22 & 56.7 & 1.68 & 53.4 & 2.11 & 50.1 & 2.58 \\
\hline $3 \mathrm{p}^{22}$ & 25.4 & 0.005 & 25.4 & 0.107 & 25.4 & 0.044 & 25.3 & 0.101 & 25.2 & 0.235 & 24.9 & 0.682 \\
\hline $3 p 4 p$ & 46.1 & 1.43 & 44.6 & 3.45 & 43.0 & 4.90 & 41.4 & 7.56 & 39.8 & 12.2 & 38.2 & 24.7 \\
\hline $3 \mathrm{p} 5 \mathrm{p}$ & 56.8 & 0.861 & 54.4 & 1.48 & 52.0 & 2.09 & 49.7 & 3.25 & 47.4 & 5.23 & 45.0 & 10.7 \\
\hline $3 \mathrm{p} 6 \mathrm{p}$ & 62.5 & 0.540 & 59.7 & 0.771 & 56.9 & 1.09 & 54.1 & 1.70 & 51.3 & 2.75 & 48.6 & 5.60 \\
\hline $3 p 7 p$ & 65.9 & 0.229 & 62.8 & 0.459 & 59.7 & 0.649 & 56.7 & 1.01 & 53.7 & 1.64 & 50.7 & 3.32 \\
\hline $3 \mathrm{p} 8 \mathrm{p}$ & 68.1 & 0.149 & 64.9 & 0.297 & 61.5 & 0.419 & 58.3 & 0.651 & 55.2 & 1.06 & 52.1 & 2.14 \\
\hline $3 \mathrm{p} 3 \mathrm{~d}$ & 27.2 & 88.5 & 27.6 & 136.0 & 27.9 & 202.0 & 28.2 & 233.3 & 28.3 & 250.0 & 28.3 & 258.0 \\
\hline $3 \mathrm{p} 4 \mathrm{~d}$ & 49.0 & 19.1 & 47.5 & 35.0 & 46.0 & 49.7 & 44.4 & 63.0 & 42.8 & 76.7 & 41.2 & 366.0 \\
\hline $3 \mathrm{p} 5 \mathrm{~d}$ & 58.6 & 7.48 & 56.2 & 16.3 & 53.9 & 20.2 & 51.5 & 26.2 & 49.2 & 32.9 & 46.8 & 28.1 \\
\hline $3 \mathrm{p} 6 \mathrm{~d}$ & 63.8 & 3.78 & 60.9 & 7.10 & 58.1 & 10.4 & 55.3 & 13.6 & 52.5 & 7.22 & 49.8 & 23.5 \\
\hline $3 \mathrm{p} 7 \mathrm{~d}$ & 66.8 & 22.1 & 63.7 & 4.21 & 60.6 & 6.13 & 57.5 & 8.47 & 54.5 & 10.3 & 51.5 & 8.45 \\
\hline $3 \mathrm{p} 8 \mathrm{~d}$ & 68.8 & 1.41 & 65.5 & 2.63 & 62.2 & 3.83 & 59.0 & 5.29 & 55.8 & 6.63 & 52.6 & 5.28 \\
\hline $3 d 4 p$ & 49.7 & 36.8 & 48.6 & 67.7 & 47.3 & 93.3 & 46.0 & 114.0 & 44.6 & 129.0 & 43.2 & 144.0 \\
\hline $3 d 5 p$ & 59.7 & 21.2 & 57.7 & 39.5 & 55.6 & 55.1 & 53.6 & 67.8 & 51.5 & 78.1 & 49.4 & 86.5 \\
\hline $3 \mathrm{~d} 6 \mathrm{p}$ & 65.0 & 13.4 & 62.5 & 25.1 & 60.1 & 51.6 & 57.6 & 43.9 & 55.1 & 50.8 & 52.6 & 56.1 \\
\hline $3 d 7 p$ & 68.1 & 8.91 & 65.4 & 1.13 & 62.7 & 23.6 & 60.0 & 29.3 & 57.2 & 32.0 & 54.5 & 37.8 \\
\hline $3 \mathrm{~d} 8 \mathrm{p}$ & 70.1 & 6.36 & 67.2 & 11.5 & 64.3 & 16.5 & 61.5 & 80.3 & 58.6 & 23.7 & 55.7 & 26.2 \\
\hline $3 d^{22}$ & 29.4 & 57.8 & 30.2 & 188.0 & 30.8 & 242.0 & 31.4 & 276.0 & 31.7 & 293.0 & 32.0 & 389.0 \\
\hline $3 d 4 d$ & 48.3 & 84.6 & 47.3 & 155.0 & 46.2 & 214.0 & 45.1 & 239.0 & 43.9 & 268.0 & 42.7 & 284.0 \\
\hline $3 \mathrm{~d} 5 \mathrm{~d}$ & 58.6 & 49.8 & 56.7 & 96.8 & 54.8 & 135.0 & 52.8 & 158.0 & 50.9 & 180.0 & 48.8 & 196.0 \\
\hline 3d6d & 64.1 & 43.6 & 61.7 & 66.7 & 59.4 & 94.2 & 57.0 & 114.0 & 54.6 & 132.0 & 25.1 & 145.0 \\
\hline $3 \mathrm{~d} 7 \mathrm{~d}$ & 67.4 & 24.2 & 64.8 & 48.0 & 62.1 & 70.0 & 59.5 & 84.9 & 56.8 & 86.4 & 54.1 & 111.0 \\
\hline $3 \mathrm{~d} 8 \mathrm{~d}$ & 68.4 & 20.2 & 66.3 & 40.0 & 63.1 & 58.3 & 59.8 & 70.8 & 58.0 & 72.0 & 55.4 & 83.7 \\
\hline
\end{tabular}

where $\left(1 / r_{12}\right)$ is the electron-electron coupling operator, and $\psi_{\mathrm{a}}$ and $\psi_{\mathrm{b}}$ are the wave functions of the active electrons.

The Einstein coefficient for a single-electron transition in a spontaneous radiation is given by

$$
A_{\mathrm{r}}^{(0)}=2.68 \times 10^{9}\left(\Delta E_{\mathrm{df}}\right)^{2}\left(\ell_{<} / 2 \ell_{\mathrm{d}}+1\right)\left|R_{\mathrm{D}}\right|^{2} .
$$

$\Delta E_{\mathrm{df}}$ in (7) must be in units of Rydberg. The radial part of the radiative matrix element $R_{\mathrm{D}}$ is defined as:

$$
R_{\mathrm{D}}=\int r^{2} \mathrm{~d} r R_{\mathrm{f}}(r) r R_{\mathrm{d}}(r)
$$

$R_{\mathrm{f}}(r)$ and $R_{\mathrm{d}}(r)$ are the radial wave functions of the orbital in final $(f)$ and resonance d-states and involved in the radiative transition.

In the present work, the DR process with $\Delta n \neq 0$ transitions, involving excitation from the $n=2$ subshell, can be represented by

$$
1 \mathrm{~s}^{2} 2 \mathrm{~s}^{2} 2 \mathrm{p}^{n}+e_{\mathrm{c}} \Leftrightarrow 1 \mathrm{~s}^{2} 2 \mathrm{~s}^{2} 2 \mathrm{p}^{n-1} \mathrm{n}_{1} \ell_{1} \mathrm{n}_{2} \ell_{2} \rightarrow
$$

Explicit calculations were carried out for the intermediate d-states with $n_{1}=3$ and $n_{2}<9$ with $\ell_{1}^{\prime}<6$.

Finally, the atomic energy levels and bound state wave functions needed in this work were calculated according to the single configuration Hartree-Fock (SCHF) model in the average level scheme.

\section{Semi-empirical Rate Formula}

Fitting of the data calculated for $\mathrm{Fe}^{Z+}$ ions was done by examining all the rates for each ion of a given $N$, where $N$ is the number of electrons in the ion before the electron capture. The $k T$ dependence of $\alpha$ at small $k T$, for which $\alpha \sim \exp [-e / k T]$ and at large $k T$ at which $\alpha \sim(k T)^{-3 / 2}$ are used in constructing the empirical rate formula. The rate coefficients for $\mathrm{Fe}^{Z+}(Z=21$, $20,19,18,17$, and 16) have been fitted to the formula $\alpha(2 p ; \Delta n \neq 0)=(A N+B)[\exp (-3.3 / k T)]\left[(k T)^{-3 / 2}\right]$,

where $A=0.844 \times 10^{-10}$ and $B=-2.805 \times 10^{-10}$. 
Table 2. The Auger $\left(A_{\mathrm{a}}\right)$ and radiative $\left(A_{\mathrm{r}}\right)$ widths of one of the intermediate states for the ions under investigation, where the radiative transtion is from $3 p$ to $2 \mathrm{~s}$. (The number in brackets is the power of ten).

\begin{tabular}{lcccccc}
\hline Ion & (i) & $e_{\mathrm{c}}(\mathrm{Ry})$ & $\ell_{\mathrm{c}}$ & $A_{\mathrm{a}}\left(\mathrm{s}^{-1}\right)$ & $(\mathrm{d})$ & $A_{\mathrm{r}}\left(\mathrm{s}^{-1}\right)$ \\
\hline $\mathrm{Fe}^{21+} 1 \mathrm{~s}^{2} 2 \mathrm{~s}^{2} 2 \mathrm{p}$ & 25.4 & 1 & $0.250(15)$ & $1 \mathrm{~s}^{2} 2 \mathrm{~s}^{2} 3 \mathrm{p}^{2}$ & $0.202(10)$ \\
& & & 3 & $0.458(13)$ & \\
$\mathrm{Fe}^{20+} 1 \mathrm{~s}^{2} 2 \mathrm{~s}^{2} 2 \mathrm{p}^{2}$ & 25.4 & 1 & $0.187(15)$ & $1 \mathrm{~s}^{22} 2 \mathrm{~s}^{22} 2 \mathrm{p} 3 \mathrm{p}^{22}$ & $0.337(11)$ \\
& & 3 & $0.356(13)$ & & \\
$\mathrm{Fe}^{19+} 1 \mathrm{~s}^{2} 2 \mathrm{~s}^{2} 2 \mathrm{p}^{3}$ & 25.4 & 1 & $0.176(15)$ & $1 \mathrm{~s}^{2} 2 \mathrm{~s}^{2} 2 \mathrm{p}^{22} 3 \mathrm{p}^{2}$ & $0.373(10)$ \\
& & 3 & $0.352(13)$ & & \\
$\mathrm{Fe}^{17+} 1 \mathrm{~s}^{2} 2 \mathrm{~s}^{2} 2 \mathrm{p}^{5}$ & 25.2 & 1 & $0.842(14)$ & $1 \mathrm{~s}^{2} 2 \mathrm{~s}^{2} 2 \mathrm{p}^{4} 3 \mathrm{p}^{2}$ & $0.592(10)$ \\
& & 3 & $0.184(13)$ & & \\
$\mathrm{Fe}^{16+} 1 \mathrm{~s}^{2} 2 \mathrm{~s}^{2} 2 \mathrm{p}^{6}$ & 24.9 & 1 & $0.409(14)$ & $1 \mathrm{~s}^{22} 2 \mathrm{~s}^{2} 2 \mathrm{p}^{5} 3 \mathrm{p}^{2}$ & $0.707(10)$ \\
& & 3 & $0.936(12)$ & & \\
\hline
\end{tabular}

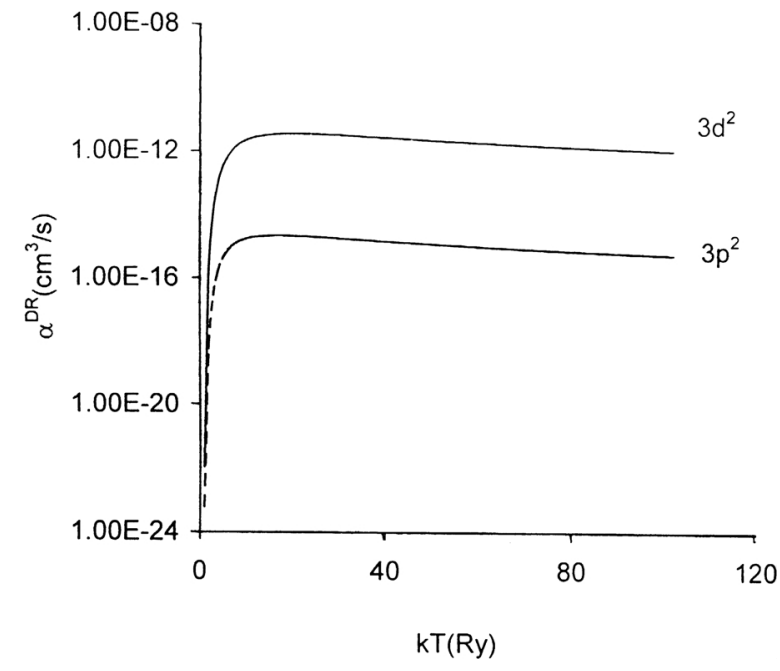

Fig. 1. Comparison between the rate coefficients of the states $3 \mathrm{p}^{2}$ and $3 \mathrm{~d}^{2}$ in case of $\mathrm{Fe}^{20+}$.

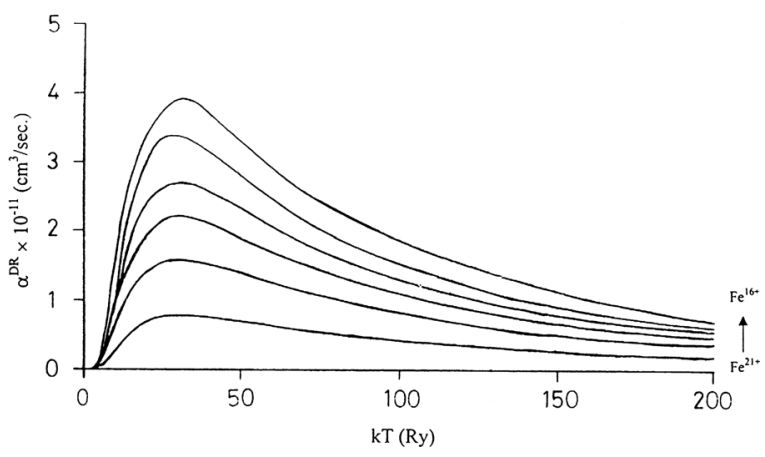

Fig. 2. The DR rate coefficients (in $\mathrm{cm}^{3} / \mathrm{s}$ ) versus $k T$ (in $\mathrm{Ry}$ ) as calculated from (5) for the ions $\mathrm{Fe}^{Z+}$ with $Z=16$ to 21 , where $\mathrm{Fe}^{21+}$ belongs to the lowest curve and $\mathrm{Fe}^{16+}$ to the highest one.

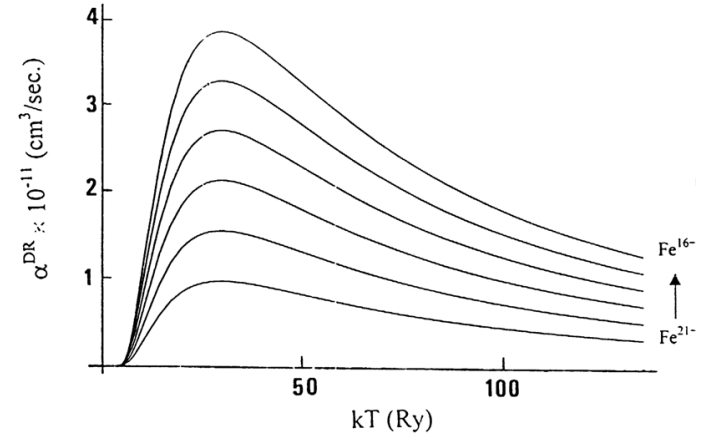

Fig. 3. The DR rate coefficients (in $\mathrm{cm}^{3} / \mathrm{s}$ ) versus $k T$ (in Ry), fitted using (10) for the same ions as in Fig. 2.

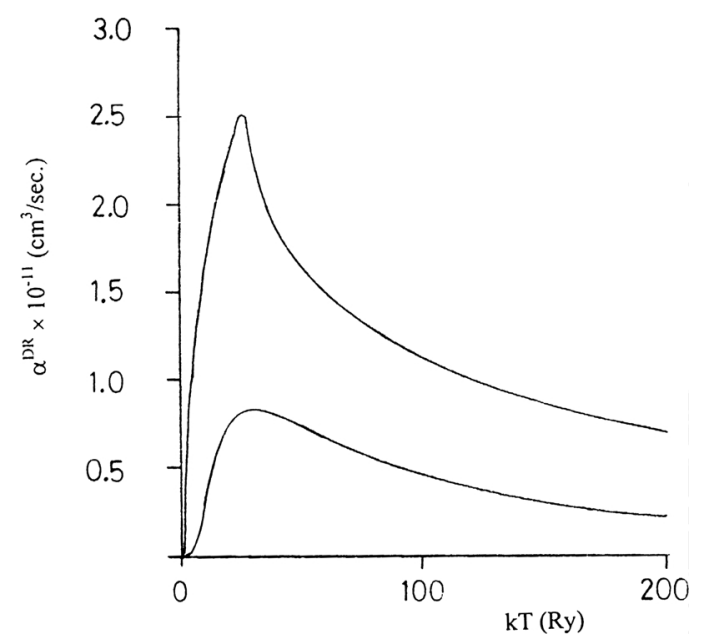

Fig. 4. Comparison between the DR rate coefficients of $\mathrm{Fe}^{21+}$ using two different empirical formulas. The upper curve is taken from Hahn [5], while the lower one is using (10) and shown also in Fig. 3.

\section{Results and Discussion}

The DR cross sections and rate coefficients for all the interesting ions are evaluated using $A_{\mathrm{a}}$ and $A_{\mathrm{r}}$. The results of the cross sections are presented in Table 1. The values of the radiative widths $A_{\mathrm{r}}$ 's are corrected for many-electron ions as in Hahn [9-11]. It is clear from Table 1 that the DR cross sections for the states 3 dnd contribute more to the rate coefficients than the other states. For example, the cross section for the state $3 \mathrm{~d}^{2}$ is $1.88 \times 10^{-19} \mathrm{~cm}^{2}$ for $\mathrm{Fe}^{20+}$, while it is $1.07 \times 10^{-22} \mathrm{~cm}^{2}$ to the state $3 \mathrm{p} 2$ for the same ion. The values of the DR cross sections given in (2) depend crucially on the $A_{\mathrm{a}}$ 's and $A_{\mathrm{r}}$ 's. Since, we have too many intermediate states, also we have a huge number of these Auger and radiative widths, therefore, we 
present some of these values in Table 2. Figure 1 shows the big difference between the contribution of the state $3 \mathrm{~d}^{2}$ and the state $3 \mathrm{p}^{2}$ to the rate coefficients in case of $\mathrm{Fe}^{20+}$. In Fig. 2 the rate coefficients for all $\mathrm{Fe}^{Z+}$ ions with $N=5,6,7,8,9$, and 10 are shown. Smooth curves were obtained. From the figure it is clear that, the rates are peaked around $k T=30 \mathrm{Ry}$. This result seems strange, and there should be a $Z$-dependence, but this change is very small, as a result of the small difference between the number of electrons in the considered Fe ions. The change is clear in Figs. 2 and 3 between $\mathrm{Fe}^{21+}$ and $\mathrm{Fe}^{16+}$. The calculated rate coefficients using (10) are shown in Fig. 3. From the figures, the agreement between the results of the empirical rate formula and the explicit calculations is obvious. Comparing between the present results with the results of Hahn [5] for $\mathrm{Fe}^{21+}$, we got a smooth curve as he got, (where the temperatures are assumed to scale as $Z^{2}$, for $\Delta n \neq 0$ ). The difference between our results and Hahn's is coming from the different modes of excitation-capture used. We used only $\Delta n \neq 0$ for 2p-excitation. This comparison is shown in Figure 4. Because of that, another empirical formula for $\Delta n=0$ is urgently needed. It should be noted that the main difficulty in constructing a simple empirical formula is the lack of reliable data. Therefore we calculated the DR

[1] A. Burgess, Astrophysics. J. 139, 776 (1964).

[2] A. L. Merts, R. D. Cowan, and N. H. Magee, LASL Report No. LA-220-MS (1976).

[3] Y. Hahn, Phys. Rev. A22, 2896 (1980).

[4] G. Omar and Y. Hahn, Z. Phys. D25, 41 (1992).

[5] Y. Hahn, JQSRT 41, 315 (1989).

[6] A. H. Moussa and Y. Hahn, JQSRT 43, 45 (1990).

[7] Y. Hahn, Comments At. Mol. Phys. 19, 99 (1987). cross sections and rate coefficients for this series of $\mathrm{Fe}$ ions. As soon as more calculations become available, and the accuracy of the data is better, this formula may be improved, perhaps to an overall accuracy of $\pm 10 \%$. The main contribution of the present work is to show that a simple and reasonable rate formula can be derived, which is free of input parameters.

\section{Conclusions}

Our results can be summarized as follows: 1.) The states 3 pnd and 3 dnd contribute more to the cross sections and rate coefficients than the other states. Therefore one can estimate the rate coefficients from these dominant states. 2.) Smooth curves were obtained for the rate coefficients for all ions. They are peaked around $k T=30 \mathrm{Ry}$. The maximum rates are scaled as $Z_{\text {eff }}$, which is not clear in the figures because of the small difference between the number of electrons in each ion. The scaling may be seen between the $\mathrm{Fe}^{21+}$ and $\mathrm{Fe}^{16+}$ ions (Fig. 2). 3.) The empirical formula (10) can successfully be used to find the rate coefficients of the $2 \mathrm{p}$-excitation for $\mathrm{Fe}^{z+}$ ions with $\Delta n \neq 0$. The main contribution of the present work is to show that a simple rate formula can be derived which is free of input parameters.

[8] H. Ramadan and Y. Hahn, Phys. Rev. 39, 3350 (1989).

[9] Y. Hahn, Adv. Atom. Molec. Phys. 21, 123 (1985).

[10] J.E. Sedley and D.F. Marran, Phys. Rev. A47, 126 (1993).

[11] H. Ramadan, Egypt. J. Phys. 33, 419 (2002).

[12] H. Ramadan and G. Omar, " Sixth Radiation Physics Conference”, Assiut, Egypt, 27 -30, Oct. (2002). 